University of Nebraska - Lincoln

DigitalCommons@University of Nebraska - Lincoln

NASA Publications

National Aeronautics and Space Administration

2010

\title{
Coupled neutron transport for HZETRN
}

T.C. Slaba

Old Dominion University, Norfolk, Tony.C.Slaba@nasa.gov

S.R. Blattnig

NASA Langley Research Center

S.K. Aghara

Prairie View A\&M University

L.W. Townsend

University of Tennessee

T. Handler

University of Tennessee

See next page for additional authors

Follow this and additional works at: https://digitalcommons.unl.edu/nasapub

Part of the Physical Sciences and Mathematics Commons

Slaba, T.C.; Blattnig, S.R.; Aghara, S.K.; Townsend, L.W.; Handler, T.; Gabriel, T.A.; Pinsky, L.S.; and Reddell, B., "Coupled neutron transport for HZETRN" (2010). NASA Publications. 87.

https://digitalcommons.unl.edu/nasapub/87

This Article is brought to you for free and open access by the National Aeronautics and Space Administration at DigitalCommons@University of Nebraska - Lincoln. It has been accepted for inclusion in NASA Publications by an authorized administrator of DigitalCommons@University of Nebraska - Lincoln. 


\section{Authors}

T.C. Slaba, S.R. Blattnig, S.K. Aghara, L.W. Townsend, T. Handler, T.A. Gabriel, L.S. Pinsky, and B. Reddell 


\title{
Coupled neutron transport for HZETRN
}

\author{
T.C. Slaba ${ }^{\mathrm{a}, *}$, S.R. Blattnig ${ }^{\mathrm{b}}$, S.K. Aghara ${ }^{\mathrm{c}}$, L.W. Townsend ${ }^{\mathrm{d}}$, T. Handler ${ }^{\text {d }}$, T.A. Gabriel ${ }^{\mathrm{e}}$, L.S. Pinsky ${ }^{\mathrm{f}}$, \\ B. Reddell ${ }^{\mathrm{f}}$
}

\author{
a Old Dominion University, Norfolk, VA 23505, USA \\ ${ }^{\mathrm{b}}$ NASA Langley Research Center, Hampton, VA 23681, USA \\ ${ }^{c}$ Prairie View A\&M University, Prairie View, TX 77446, USA \\ ${ }^{\mathrm{d}}$ University of Tennessee, Knoxville, TN 37996, USA \\ e Scientific Investigation and Development, Knoxville, TN 37922, USA \\ ${ }^{\mathrm{f}}$ University of Houston, Houston, TX 77204, USA
}

\section{A R T I C L E I N F O}

\section{Article history:}

Received 21 November 2008

Received in revised form

26 October 2009

Accepted 5 January 2010

\section{Keywords:}

Neutron transport

Radiation constraints

Space radiation

Dose

Space radiation transport

HZETRN

\begin{abstract}
A B S T R A C T
Exposure estimates inside space vehicles, surface habitats, and high altitude aircrafts exposed to space radiation are highly influenced by secondary neutron production. The deterministic transport code HZETRN has been identified as a reliable and efficient tool for such studies, but improvements to the underlying transport models and numerical methods are still necessary. In this paper, the forwardbackward (FB) and directionally coupled forward-backward (DC) neutron transport models are derived, numerical methods for the FB model are reviewed, and a computationally efficient numerical solution is presented for the DC model. Both models are compared to the Monte Carlo codes HETC-HEDS, FLUKA, and MCNPX, and the DC model is shown to agree closely with the Monte Carlo results. Finally, it is found in the development of either model that the decoupling of low energy neutrons from the light ion transport procedure adversely affects low energy light ion fluence spectra and exposure quantities. A first order correction is presented to resolve the problem, and it is shown to be both accurate and efficient.
\end{abstract} (C) 2010 Elsevier Ltd. All rights reserved.

\section{Introduction}

Radiation exposure guidelines are a primary concern for the design of personal shielding, spacecraft, instrumentation, and mission planning. Consequently, there is significant interest in developing computational tools that allow shield analyses not only in simplified geometries, but in more complex final design models as well (Wilson et al., 2003). The deterministic transport code HZETRN (Wilson and Badavi 1986; Wilson et al., 1991, 2003, 2006; Cucinotta, 1993; Shinn et al., 1991), developed at NASA Langley Research Center has emerged in recent years as a reliable and efficient tool for such studies. It has shown reasonable accuracy in deep space galactic cosmic ray (GCR), solar particle event (SPE), and low earth orbit (LEO) simulations when compared to either Monte Carlo results or experimental data (Wilson et al., 2005). However, such verification and validation has revealed a deficiency in the low energy neutron transport procedure (Shinn et al., 1994). HZETRN utilizes the straight ahead approximation in which all fragments are assumed to propagate in the same direction as the projectile. The assumption is accurate for high energy charged particles but

\footnotetext{
* Corresponding author. Tel.: +1 7578641420 .

E-mail address: Tony.C.Slaba@nasa.gov (T.C. Slaba).
}

breaks down for low energy neutrons which are produced nearly isotropically (Alsmiller et al., 1965). This is significant for heavily shielded space vehicles, surface habitats, and high altitude aircraft where secondary neutron production is important in exposure calculations (Getselev et al., 2004).

Several neutron transport models have been developed for HZETRN, with recent efforts focused on identifying an optimal bi-directional neutron transport model and solution method. The terms model and method are used extensively throughout this paper; model is used to refer to the set of governing transport equations, while method is used to refer to the analytic or numerical techniques used to solve a model. Heinbockel et al. (2000) and Clowdsley et al. (2000a,b) developed the forwardbackward (FB) neutron transport model. It assumes that low energy neutrons can be split into forward and backward components, and multiple reflections from forward to backward (or vice versa) can be ignored. Feldman (2003) expanded on the work of Heinbockel and Clowdsley by developing the directionally coupled forwardbackward (DC) neutron transport model. It also assumes that low energy neutrons can be split into forward and backward components, but multiple reflections are accounted for in the governing transport equations. The numerical methods used for each model were significantly different as well. 
Heinbockel et al. (2000) and Clowdsley et al. (2000a,b) used a multigroup method for solving the FB model. Multigroup methods have a long history in nuclear reactor theory and assume that fluences or cross sections are constant over small regions, or groups, of the energy spectrum (Marchuk and Lebedev, 1986). Marchuk and Lebedev (1986) gives a more general description of this method. Feldman (2003) used a collocation technique along with first order finite differencing for the DC model. Collocation and finite differencing methods use polynomial expansions of the solution to transform a differential equation into a system of linear equations (Deuflhard and Bornemann, 2002). In this case, the system was sufficiently large that it had to be solved numerically. Deuflhard and Bornemann (2002) and Demmel (1997) give more detailed descriptions of these numerical methods.

Recently, Slaba et al. (2006), Slaba (2007) and Heinbockel et al. (2007) developed three methods for solving the FB model which they called the collocation method, the fixed-point series method, and Wilson's method. The latter two methods are both based on Neumann series solutions wherein each term of the series solves a simple set of equations related to the original model. The work allowed for intensive verification of the multigroup method, and the collocation method was identified as the most accurate and computationally efficient (Slaba, 2007). Slaba (2007) also indicated that a combination of the methods could be used to obtain a computationally efficient Neumann series solution to the DC model.

Finally, despite all of the attention given to neutron transport models and methods, accurately coupling these models back into HZETRN remained unresolved. The impact of such a coupling has not been previously studied in detail and must be examined if any of the neutron transport models are to be used in design studies.

In this paper, we first give a summary of the neutron transport models and methods developed thus far and present an efficient method for solving the DC model. Comparisons are made between the FB and DC models and the Monte Carlo codes HETC-HEDS (Gabriel et al., 1995; Townsend et al., 2005), FLUKA (Fasso et al., 2003, 2005), and MCNPX (Briesmeister, 2000; MCNPX 2.6.0 Manual, 2008). The impact of decoupling low energy neutrons from the light ion transport procedure in HZETRN is also examined, and a first order correction is presented. Fluence spectra and dose quantities are given to exhibit the accuracy of the proposed correction.

\section{HZETRN description}

The one-dimensional Boltzmann transport equation for charged and neutral particles with the continuous slowing down and straight ahead approximations is given as (Wilson et al., 1991)

$B\left[\phi_{j}\right]=\sum_{k} \int_{E}^{\infty} \sigma_{j k}\left(E, E^{\prime}\right) \phi_{k}\left(x, E^{\prime}\right) \mathrm{d} E^{\prime}$

with the linear differential operator

$B\left[\phi_{j}\right] \equiv\left[\frac{\partial}{\partial x}-\frac{1}{A_{j}} \frac{\partial}{\partial E} S_{j}(E)+\sigma_{j}(E)\right] \phi_{j}$

where $\phi_{j}$ is the fluence of type $j$ ions at depth $x$ with kinetic energy $E$ $(\mathrm{AMeV}), A_{j}$ is the atomic mass of a type $j$ particle, $S_{j}(E)$ is the stopping power of a type $j$ ion with kinetic energy $E, \sigma_{j}(E)$ is the total macroscopic cross section for a type $j$ particle with kinetic energy $E$, and $\sigma_{j k}\left(E, E^{\prime}\right)$ is the macroscopic production cross section for interactions in which a type $k$ particle with kinetic energy $E^{\prime}$ produce a type $j$ particle with kinetic energy $E$. Macroscopic cross sections are obtained by multiplying the corresponding microscopic cross section by the target particle mass density (Wilson et al., 1991). Hereafter, it is assumed that all cross sections are macroscopic whether it is explicitly written or not.

Wilson and Badavi 1986; Wilson et al. (1991, 2003, 2006; Cucinotta, 1993; Shinn et al., 1991) obtained approximate solutions to equation (1) by introducing the scaled quantities

$\psi_{j}(x, r)=\nu_{j} S_{p}(E) \phi_{j}(x, E)$

$s_{j k}\left(r, r^{\prime}\right)=S_{p}(E) \sigma_{j k}\left(E, E^{\prime}\right)$,

where $S_{p}(E)$ is the proton stopping power, $r$ is the residual proton range

$r=\int_{0}^{E} \frac{\mathrm{d} E^{\prime}}{S_{p}\left(E^{\prime}\right)}$

and the scaling parameter $\nu_{j}=Z_{j}^{2} / A_{j}$. For neutrons, $\nu$ is taken as unity in fluence scaling relations and zero in range scaling relations. This will be explained in more detail shortly. Equation (1) is now given in terms of the variables $x$ and $r$ as

$$
\left[\frac{\partial}{\partial x}-\nu_{j} \frac{\partial}{\partial r} S_{p}(E)+\sigma_{j}(r)\right] \psi_{j}(x, r)=\sum_{k} \frac{\nu_{j}}{\nu_{k}} \int_{r}^{\infty} s_{j k}\left(r, r^{\prime}\right) \psi_{k}\left(x, r^{\prime}\right) \mathrm{d} r^{\prime}
$$

which can be inverted using the method of characteristics (Haberman, 1998) and written as the Volterra type integral equation (Wilson et al., 2006)

$$
\begin{aligned}
\psi_{j}(x, r)= & e^{-\varsigma_{j}(r, x)} \psi_{j}\left(0, r+\nu_{j} x\right) \\
& +\sum_{k} \frac{\nu_{j}}{\nu_{k}} \int_{0}^{x} \int_{r+\nu_{j} x^{\prime}}^{\infty} e^{-\varsigma_{j}\left(r, x^{\prime}\right)} s_{j k}\left(r+\nu_{j} x^{\prime}, r^{\prime}\right) \psi_{k}\left(x-x^{\prime}, r^{\prime}\right) \mathrm{d} r^{\prime} \mathrm{d} x^{\prime}
\end{aligned}
$$

with

$\varsigma_{j}(r, x)=\int_{0}^{x} \sigma_{j}\left(r+\nu_{j} t\right) \mathrm{d} t$

Note that $\nu_{j}$ in equation (3) and $\nu_{j} / \nu_{k}$ in equation (6) are both fluence scaling relations, and $\nu_{n}=1$ in both cases to provide a nontrivial scaling (see equation (3)). Conversely, wherever $\nu$ appears as the argument of a fluence or cross section in equations (7) and (8), it is a range scaling relation and $\nu_{n}=0$. This convention is taken to reflect the absence of atomic interactions in neutron transport $\left(S_{n}(E) \equiv 0\right)$.

From here, the problem is split into two parts: heavy ions $(A>4)$ and light particles $(A \leq 4)$. For heavy ions, it is noted that projectile fragments have energy and direction very near that of the projectile, while target fragments are produced nearly isotropically with low energy and travel only a short distance before being absorbed. This approximate decoupling of target and projectile fragments is discussed in detail by Wilson et al. (1991) and suggests that heavy target fragments can be neglected in the heavy ion transport procedure (their contribution to dose is approximately accounted for after the transport procedure). The production cross section can now be recast as

$s_{j k}\left(r, r^{\prime}\right)=\sigma_{j k}(r) \delta\left(r-r^{\prime}\right)$. 
Equation (9) physically describes the nearly equal energy (or velocity) of the projectile and secondary particles. The final marching procedure for heavy ions was developed by Wilson et al. (2006) as

$$
\begin{aligned}
\psi_{j}(x+h, r)= & e^{-\zeta_{j}(r, h)} \psi_{j}\left(x, r+\nu_{j} h\right)+\sum_{k>j} \frac{\nu_{j}}{\nu_{k}} \sigma_{j k}\left(r+\nu_{j} \frac{h}{2}\right) \\
& \times \psi_{k}\left(x, r+\left(\nu_{j}+\nu_{k}\right) \frac{h}{2}\right) \\
& \times\left[\frac{e^{-\sigma_{j}\left(r+\nu_{j}\right) h}-e^{-\sigma_{k}\left(r+\left(\nu_{j}+\nu_{k}\right) \frac{h}{2}\right) h}}{\sigma_{k}\left(r+\left(\nu_{j}+\nu_{k}\right) \frac{h}{2}\right)-\sigma_{j}\left(r+\nu_{j} \frac{h}{2}\right)}\right]+O\left(h^{2}\right)
\end{aligned}
$$

where $h$ is the step size. For light particles, both projectile and target fragments are included in the transport procedure. The broad energy distribution in collision events also indicates that equation (9) cannot be used. The final marching procedure for light particles was also developed by Wilson et al. (1986, 1991, 2003, 2006); (Cucinotta, 1993); Shinn et al. (1991) as

$$
\begin{aligned}
\psi_{j}(x+h, r)= & e^{-\zeta_{j}(r, h)} \psi_{j}\left(x, r+\nu_{j} h\right)+\sum_{k} \frac{\nu_{j}}{\nu_{k}} \int_{r+\nu_{j} h / 2}^{\infty} e^{-\zeta_{j}\left(r, \frac{h}{2}\right)-\zeta_{k}\left(r^{\prime}, \frac{h}{2}\right)} \\
& \times F_{j k}^{\Delta}\left(r, r^{\prime} ; h\right) \psi_{k}\left(x, r^{\prime}+\nu_{k} \frac{h}{2}\right) \mathrm{d} r^{\prime}+O\left(h^{2}\right),
\end{aligned}
$$

where the integrand has been simplified using

$F_{j k}^{\Delta}\left(r, r^{\prime} ; h\right)=\int_{0}^{h} s_{j k}\left(r+\nu_{j} z, r^{\prime}\right) \mathrm{d} z$.

The nature of the boundary condition, or environment, determines how equation (10) and (11) are evaluated and coupled. For most SPE environments, there are a negligible number of heavy ion projectiles, and only equation (11) is evaluated. For GCR environments, equation (10) is evaluated for heavy ions. equation (11) is evaluated for light particles, and the summation term appearing in equation (10) is added. The summation is taken only over heavy ion projectiles, and it physically accounts for light ion production from heavy ion projectiles.

The marching equations (10) and (11) will be referred to throughout this paper as the HZETRN marching algorithm, with the proper equations evaluated and coupled for a given environment. It is important to note also that all particles, including neutrons, are propagated in the straight ahead direction in the HZETRN marching algorithm.

\section{Neutron transport models}

To derive the neutron transport models, we first decompose the particle fluence and neutron production cross section into straight ahead and isotropic components. This approximation is justified by Wilson et al. (1991) who showed that projectile fragments are produced with energy and direction very near that of the projectile, while target fragments are produced nearly isotropically with very low energy. For particle fluences, the decomposition is given simply as (Wilson et al., 2005)

$\phi_{j}(x, E)=\phi_{j}^{i s o}(x, E)+\phi_{j}^{s a}(x, E)$.
To decompose the neutron production cross section, we first distinguish between the nuclear reactive and elastic parts

$\sigma_{n k}\left(E, E^{\prime}\right)=\sigma_{n k}^{r}\left(E, E^{\prime}\right)+\sigma_{n k}^{e l}\left(E, E^{\prime}\right)$,

where $\sigma_{n k}^{e l}\left(E, E^{\prime}\right)=0$ for $k \neq n$. The angularly dependent nuclear reactive neutron production cross section can be represented as (Wilson et al., 2005)

$\widehat{\sigma}_{n k}^{r}\left(E, E^{\prime}, \mathbf{\Omega}, \mathbf{\Omega}^{\prime}\right)=g\left(A_{T}, E, \theta\right) \sigma_{n k}^{r}\left(E, E^{\prime}\right)$,

with (Ranft, 1980)

$g\left(A_{T}, E, \theta\right)=\left\{\begin{array}{cc}N e^{-\theta^{2} / \lambda}, & 0 \leq \theta \leq \pi / 2 \\ N e^{-\pi^{2} / 4 \lambda}, & \pi / 2<\theta \leq \pi\end{array}\right.$,

and $\lambda=\left(120+0.36 A_{T}\right) / E$, where $E$ is the produced neutron kinetic energy in $\mathrm{MeV}, A_{T}$ is the atomic mass of the target nucleus, $\theta$ is the production angle with respect to the direction of propagation, and $N$ is a normalization constant such that

$2 \pi \int_{0}^{\pi} g\left(A_{T}, E, \theta\right) \mathrm{d} \theta=1$.

The parameterization constants, 120 and 0.36 have units $\mathrm{MeV}$ and $\mathrm{MeV} / \mathrm{amu}$, respectively, so that $\lambda$ is dimensionless.

The nuclear reactive neutron production cross section can now be decomposed into forward (scattering angle $\theta \in[0, \pi / 2]$ ) and backward (scattering angle $\theta \in(\pi / 2, \pi])$ components

$$
\begin{aligned}
\sigma_{n k}^{r}\left(E, E^{\prime}\right) & =F^{(+)}\left(A_{T}, E\right) \sigma_{n k}^{r}\left(E, E^{\prime}\right)+F^{(-)}\left(A_{T}, E\right) \sigma_{n k}^{r}\left(E, E^{\prime}\right) \\
& \equiv \sigma_{n k}^{r,(+)}\left(E, E^{\prime}\right)+\sigma_{n k}^{r,(-)}\left(E, E^{\prime}\right)
\end{aligned}
$$

where the forward and backward coefficients are

$$
\begin{aligned}
& F^{(+)}\left(A_{T}, E\right)=\int_{0}^{\pi / 2} g\left(A_{T}, E, \theta\right) \mathrm{d} \theta \\
& F^{(-)}\left(A_{T}, E\right)=1-F^{(+)}\left(A_{T}, E\right) .
\end{aligned}
$$

The straight ahead and isotropic coefficients are therefore defined as (Clowdsley et al., 2000b)

$$
\begin{aligned}
& F^{s a}\left(A_{T}, E\right)=1-F^{i s o}\left(A_{T}, E\right) \\
& F^{i s o}\left(A_{T}, E\right)=2 F^{(-)}\left(A_{T}, E\right),
\end{aligned}
$$

so that the nuclear reactive neutron production cross section can be expressed in terms of isotropic and straight ahead components

$$
\begin{aligned}
\sigma_{n k}^{r}\left(E, E^{\prime}\right) & =F^{s a}\left(A_{T}, E\right) \sigma_{n k}^{r}\left(E, E^{\prime}\right)+F^{i s o}\left(A_{T}, E\right) \sigma_{n k}^{r}\left(E, E^{\prime}\right) \\
& \equiv \sigma_{n k}^{r, s a}\left(E, E^{\prime}\right)+\sigma_{n k}^{r, i s o}\left(E, E^{\prime}\right) .
\end{aligned}
$$

The decompositions in equations (18) and (23) are depicted in Figs. 1 and 2 for a $500 \mathrm{MeV}$ proton projectile and an Aluminum target. It should be noted in Fig. 2 that the straight ahead component of the nuclear reactive neutron production cross section is dominant at high fragment energies, while the isotropic component is dominant at low fragment energies as suggested earlier.

Equations (13), (14) and (23) are substituted into equation (1), and the HZETRN marching algorithm is used to solve 


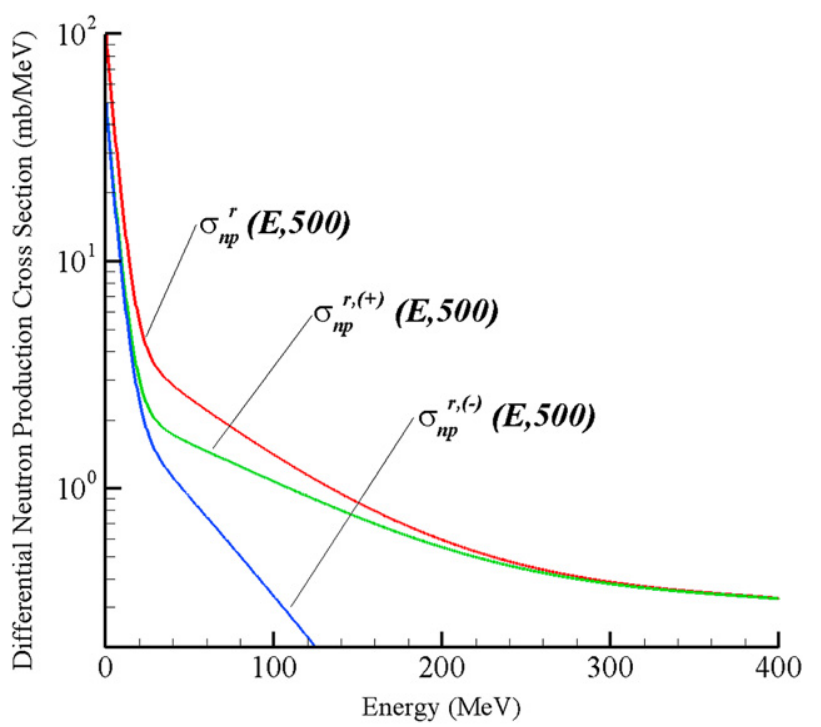

Fig. 1. Forward, backward, and total neutron nuclear reactive production cross section for a $500 \mathrm{MeV}$ proton projectile and Aluminum target.

$B\left[\phi_{j}^{s a}\right]=\sum_{k} \int_{E}^{\infty} \kappa_{j k}^{s a}\left(E, E^{\prime}\right) \phi_{k}^{s a}\left(x, E^{\prime}\right) \mathrm{d} E^{\prime}$,

where the production cross section $\kappa_{j k}\left(E, E^{\prime}\right)$ is piecewise defined as

$\kappa_{j k}^{s a}\left(E, E^{\prime}\right)=\left\{\begin{array}{cc}\sigma_{n k}^{r, s a}\left(E, E^{\prime}\right)+\sigma_{n k}^{e l}\left(E, E^{\prime}\right), & j=n \\ \sigma_{j k}\left(E, E^{\prime}\right), & j \neq n\end{array}\right.$,

The only difference between equations (1) and (24) is the form of the neutron production cross section; equation (1) uses the total nuclear reactive neutron production cross section, while equation (24) uses only the straight ahead component. It is clear from Figs. 2 and 3 that the impact of using only the straight ahead component of the nuclear reactive neutron production cross section will be a reduced or under-predicted neutron fluence spectrum below

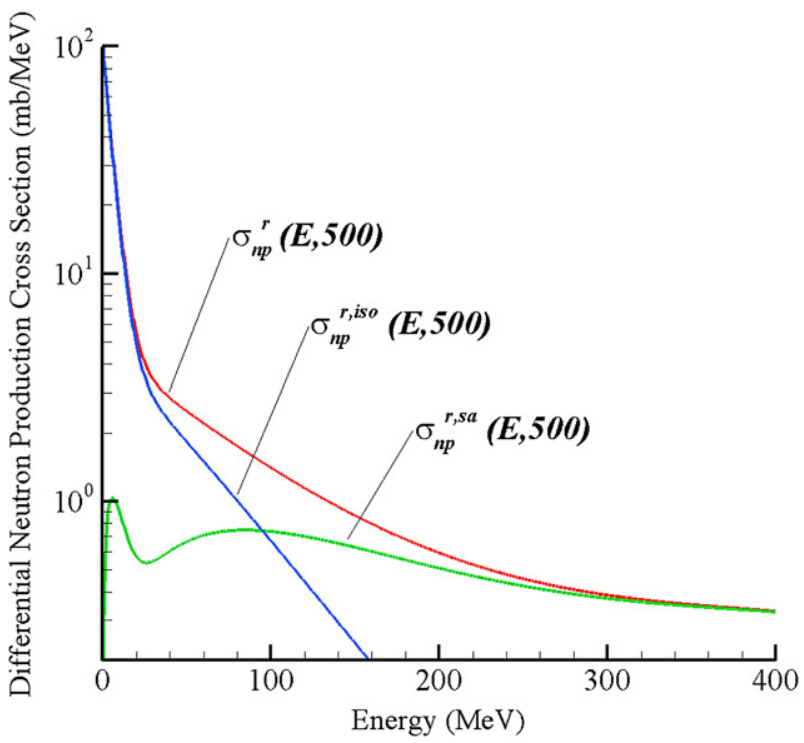

Fig. 2. Straight ahead, isotropic, and total neutron nuclear reactive production cross section for a $500 \mathrm{MeV}$ proton projectile and Aluminum target.

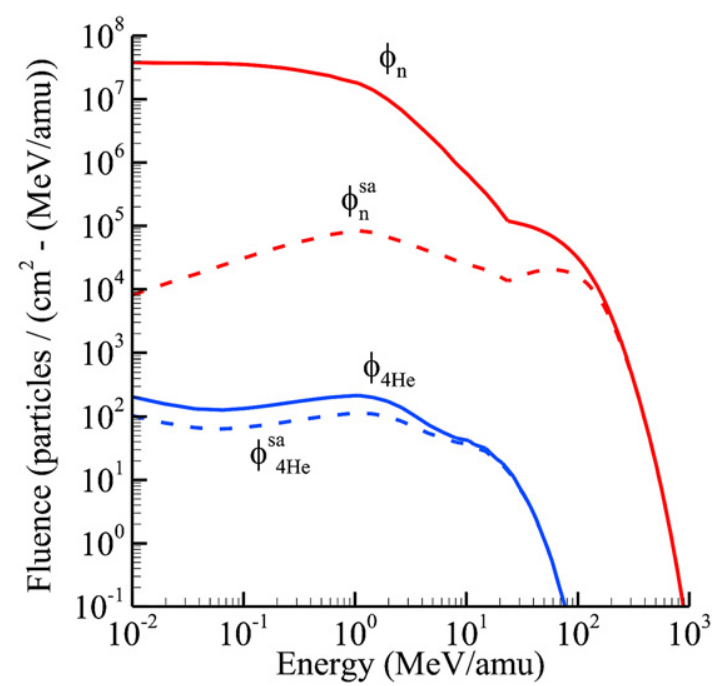

Fig. 3. Neutron and ${ }^{4} \mathrm{He}$ fluence spectra at $50 \mathrm{~g} / \mathrm{cm}^{2}$ in an Aluminum target exposed to the February, 1956 Webber (Quenby and Webber, 1959) SPE.

$\sim 200 \mathrm{MeV}$. An examination of the HZETRN marching algorithm indicates that a residual under-prediction will occur in the low energy light ion spectra as well. More will be said about this later in the paper.

We are now left to solve

$$
\begin{aligned}
B\left[\phi_{n}^{i s o}\right]= & \sum_{k \neq n} \int_{E}^{\infty} \sigma_{n k}\left(E, E^{\prime}\right) \phi_{k}^{i s o}\left(x, E^{\prime}\right) \mathrm{d} E^{\prime} \\
& +\int_{E}^{\infty} \sigma_{n n}\left(E, E^{\prime}\right) \phi_{n}^{i s o}\left(x, E^{\prime}\right) \mathrm{d} E^{\prime}+\eta_{n}(x, E),
\end{aligned}
$$

with the isotropic neutron source term

$\eta_{n}(x, E)=\sum_{k} \int_{E}^{\infty} \sigma_{n k}^{r, i s o}\left(E, E^{\prime}\right) \phi_{k}^{s a}\left(x, E^{\prime}\right) \mathrm{d} E^{\prime}$.

The isotropic component of the charged particles is obtained by solving

$B\left[\phi_{j}^{i s o}\right]=\sum_{k} \int_{E}^{\infty} \sigma_{j k}\left(E, E^{\prime}\right) \phi_{k}^{i s o}\left(x, E^{\prime}\right) \mathrm{d} E^{\prime}$,

which will be handled later. For now, we return to equation (26) and note that the sum over particle type is dropped since the range of a low energy charged particle is much shorter than its nuclear mean free path length (Wilson et al., 1991) as seen in Fig. 4.

In this case, the isotropic neutron transport equation reduces to

$B\left[\phi_{n}^{i s o}\right]=\int_{E}^{\infty} \sigma_{n n}\left(E, E^{\prime}\right) \phi_{n}^{i s o}\left(x, E^{\prime}\right) \mathrm{d} E^{\prime}+\eta_{n}(x, E)$.

The isotropic neutron fluence is now decomposed into forward and backward components according to

$\phi_{n}^{i s o}(x, E)=\phi_{n}^{f}(x, E)+\phi_{n}^{b}(x, E)$.

The terms forward and backward have different meanings when applied to fluences and cross sections. The forward component of the isotropic neutron fluence refers to all neutrons propagating in 


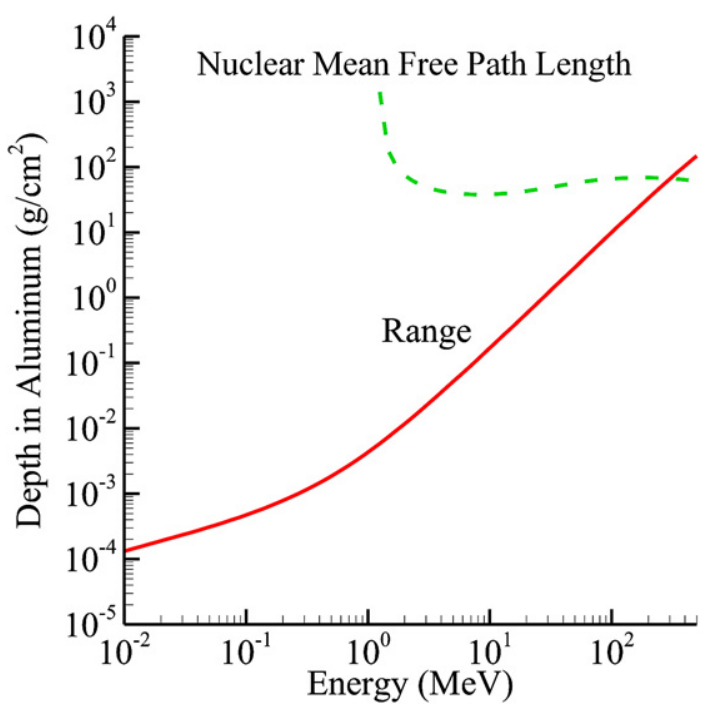

Fig. 4. Range and nuclear mean free path length of ${ }^{4} \mathrm{He}$ in Aluminum.

the forward direction; the backward component of the isotropic neutron fluence refers to all neutrons propagating in the backward direction. The forward component of the neutron production cross section refers to those neutrons produced with scattering angle in the interval $[0, \pi / 2]$ (no change in direction of propagation); the backward component of the neutron production cross section refers to those neutrons produced with scattering angle in the interval $[\pi / 2, \pi]$ (change in direction of propagation from forward to backward or vice versa).

The nuclear reactive neutron production cross section is assumed to take the form of equation (18), thereby splitting it into forward and backward components. The elastic portion is also decomposed, but it is accomplished by considering the relationship between scattering angle, pre-collision energy $\left(E^{\prime}\right)$ and post-collision energy $(E)$ given by Haffner (1967)

$E=E^{\prime}\left[\frac{A_{T}^{2}+2 A_{T} \cos \theta+1}{\left(A_{T}+1\right)^{2}}\right]$.

Forward scattering occurs for $\theta \in[0, \pi / 2]$ or for $E^{\prime} \in[E, E / \beta]$, and backward scattering occurs for $\theta \in(\pi / 2, \pi]$ or for $E^{\prime} \in(E / \beta, E / \alpha]$ where

$\alpha=\left(\frac{A_{T}-1}{A_{T}+1}\right)^{2}$

$\beta=\frac{A_{T}^{2}+1}{\left(A_{T}+1\right)^{2}}$

The neutron elastic production cross sections are then decomposed into forward and backward components

$\sigma_{n n}^{e l,(+)}\left(E, E^{\prime}\right)=\left\{\begin{array}{c}\sigma_{n n}^{e l}\left(E, E^{\prime}\right), E^{\prime} \in[E, E / \beta] \\ 0, \text { otherwise }\end{array}\right.$

$\sigma_{n n}^{e l,(-)}\left(E, E^{\prime}\right)=\left\{\begin{array}{c}\sigma_{n n}^{e l}\left(E, E^{\prime}\right), E^{\prime} \in(E / \beta, E / \alpha] \\ 0, \text { otherwise }\end{array}\right.$

The complete neutron production cross section can now be expressed as forward and backward components according to

$\sigma_{n n}\left(E, E^{\prime}\right)=\sigma_{n n}^{(+)}\left(E, E^{\prime}\right)+\sigma_{n n}^{(-)}\left(E, E^{\prime}\right)$,

with

$$
\begin{aligned}
& \sigma_{n n}^{(+)}\left(E, E^{\prime}\right)=\sigma_{n n}^{e l,(+)}\left(E, E^{\prime}\right)+\sigma_{n n}^{r,(+)}\left(E, E^{\prime}\right) \\
& \sigma_{n n}^{(-)}\left(E, E^{\prime}\right)=\sigma_{n n}^{e l,(-)}\left(E, E^{\prime}\right)+\sigma_{n n}^{r,(-)}\left(E, E^{\prime}\right) .
\end{aligned}
$$

From Feldman (2003), substitution of equations (30) and (36) into equation (29) results in the DC neutron transport model

$$
\begin{aligned}
B^{(+)}\left[\phi_{n}^{f}\right]= & \int_{E}^{\infty} \sigma_{n n}^{(+)}\left(E, E^{\prime}\right) \phi_{n}^{f}\left(x, E^{\prime}\right) \mathrm{d} E^{\prime}+\int_{E}^{\infty} \sigma_{n n}^{(-)}(E, E \prime) \phi_{n}^{b}\left(x, E^{\prime}\right) \mathrm{d} E^{\prime} \\
& +\eta_{n}^{f}(x, E) \\
B^{(-)}\left[\phi_{n}^{b}\right]= & \int_{E}^{\infty} \sigma_{n n}^{(+)}\left(E, E^{\prime}\right) \phi_{n}^{b}\left(x, E^{\prime}\right) \mathrm{d} E^{\prime}+\int_{E}^{\infty} \sigma_{n n}^{(-)}\left(E, E^{\prime}\right) \phi_{n}^{f}\left(x, E^{\prime}\right) \mathrm{d} E^{\prime} \\
& +\eta_{n}^{b}(x, E),
\end{aligned}
$$

with the source terms $\eta_{n}^{f}(x, E)$ and $\eta_{n}^{b}(x, E)$ each taken to be one half of the isotropic source term $\eta_{n}(x, E)$, and the linear differential operators are

$$
\begin{aligned}
& B^{(+)}[\phi]=\left[\frac{\partial}{\partial x}+\sigma_{n}(E)\right] \phi(x, E) \\
& B^{(-)}[\phi]=\left[-\frac{\partial}{\partial x}+\sigma_{n}(E)\right] \phi(x, E) .
\end{aligned}
$$

Note that the minus sign in equation (42) accounts for a directional change in propagation. In equation (39), the second integral represents neutrons produced in the forward direction by backward propagating neutrons. In equation (40), the second integral represents neutrons produced in the backward direction by forward propagating neutrons.

Slaba (2007) then showed that by making the simple approximation $\phi_{n}^{f}(x, E) \approx \phi_{n}^{b}(x, E)$ one obtains

$$
B^{(+)}\left[\phi_{n}^{f}\right]=\int_{E}^{\infty} \sigma_{n n}\left(E, E^{\prime}\right) \phi_{n}^{f}\left(x, E^{\prime}\right) \mathrm{d} E^{\prime}+\eta_{n}^{f}(x, E)
$$

$B^{(-)}\left[\phi_{n}^{b}\right]=\int_{E}^{\infty} \sigma_{n n}\left(E, E^{\prime}\right) \phi_{n}^{b}\left(x, E^{\prime}\right) \mathrm{d} E^{\prime}+\eta_{n}^{b}(x, E)$.

Equations (43) and (44) define the FB neutron transport model. The physical difference between the models can be deduced by examining the collision integrals. The DC model accounts for multiple changes of direction (from forward to backward or vice versa) in the coupling source integrals, while the FB model only accounts for a single change of direction. That is, after the initial decomposition of the isotropic neutron source term into forward and backward components, it is assumed in the FB model that all forward neutrons propagate forward, and all backward neutrons propagate backward. The two models will be compared later in the paper.

\section{FB Neutron transport methods}

Heinbockel et al. (2000) and Clowdsley et al. (2000a,b) used a multigroup method for solving the FB neutron transport model. The computational procedure is developed by partitioning the 
energy grid with respect to the elastic interaction parameter $\alpha$ defined in equation (32). Equations (43) and (44) are then integrated with respect to energy for some discrete set of energy values. The order of integration is switched in the collision integral and a mean value theorem is applied to evaluate the integrals. The result is an upper triangular system of first order ordinary differential equations solved using back substitution. Though the computational procedure turned out to be relatively efficient, the target dependent energy grid made energy grid convergence testing difficult, and selection of the free parameter in a mean value theorem came down to trial and error for several materials.

(Slaba et al., 2006)), Slaba (2007) and Heinbockel et al. (2007) developed three different methods (collocation method, fixedpoint series method, Wilson's method) for solving the FB model and found them to be in good agreement with the multigroup technique. The collocation method (Slaba, 2007; Heinbockel et al., 2007) is implemented by assuming that forward and backward neutron fluences can be expressed as a sum of linear basis splines

$\psi_{n}^{f}(x, E)=\sum_{j=1}^{N} B_{j}(E) \phi_{n}^{f}\left(x, E_{j}\right)$

$\psi_{n}^{b}(x, E)=\sum_{j=1}^{N} B_{j}(E) \phi_{n}^{b}\left(x, E_{j}\right)$

with

$B_{j}(E)=\left\{\begin{array}{cc}\left(E-E_{j-1}\right) /\left(E_{j}-E_{j-1}\right), & E \in\left[E_{j-1}, E_{j}\right] \\ \left(E-E_{j+1}\right) /\left(E_{j+1}-E_{j}\right), & E \in\left(E_{j}, E_{j+1}\right] \\ 0, \text { otherwise }\end{array}\right.$

The approximations are substituted into the FB model resulting in two upper triangular systems of first order linear ordinary differential equations. Using back substitution, the analytic solution to the systems are obtained (Slaba, 2007; Heinbockel et al., 2007)

$$
\begin{aligned}
\phi_{n}^{f}\left(x, E_{N-j}\right)= & e^{a_{N-j, N-j}(x)} \phi_{n}^{f}\left(0, E_{N-j}\right) \\
& +\int_{0}^{x} e^{a_{N-j, N-j}(x-x \prime)} \eta_{n}^{f}\left(x \prime, E_{N-j}\right) \mathrm{d} x \prime+\left(1-\delta_{j 0}\right) \\
& \times \sum_{k=0}^{j-1} a_{N-j, N-k} \int_{0}^{x} e^{a_{N-j, N-j}(x-\chi \prime)} \phi_{n}^{f}\left(x \prime, E_{N-k}\right) \mathrm{d} x \prime,
\end{aligned}
$$

$$
\begin{aligned}
\phi_{n}^{b}\left(x, E_{N-j}\right)= & e^{a_{N-j, N-j}(L-x)} \phi_{n}^{b}\left(L, E_{N-j}\right) \\
& +\int_{x}^{L} e^{a_{N-j, N-j}\left(x^{\prime}-x\right)} \eta_{n}^{b}\left(x^{\prime}, E_{N-j}\right) \mathrm{d} x^{\prime}+\left(1-\delta_{j 0}\right) \\
& \times \sum_{k=0}^{j-1} a_{N-j, N-k} \int_{x}^{L} e^{a_{N-j, N-j}\left(x^{\prime}-x\right)} \phi_{n}^{b}\left(x^{\prime}, E_{N-k}\right) \mathrm{d} x^{\prime},
\end{aligned}
$$

where $\delta$ is the Kronecker delta, $L$ is the thickness of the material, and the coefficients $\left\{a_{i j}\right\}$ are defined

$a_{i j}=-\sigma_{n}\left(E_{i}\right) B_{j}\left(E_{i}\right)+\int_{E_{i}}^{\infty} \sigma_{n n}\left(E_{i}, E^{\prime}\right) B_{j}\left(E^{\prime}\right) \mathrm{d} E^{\prime}$.

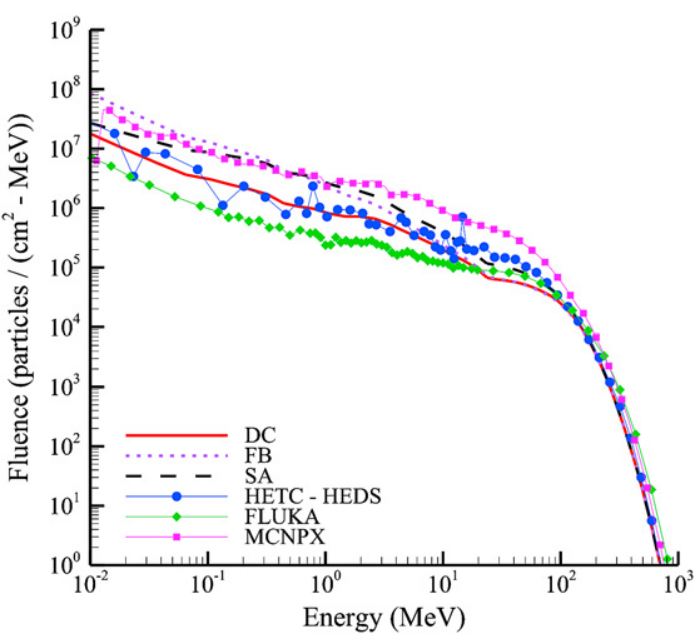

Fig. 5. Forward neutron fluence at $20 \mathrm{~g} / \mathrm{cm}^{2}$ in a $30 \mathrm{~g} / \mathrm{cm}^{2}$ water target behind a $20 \mathrm{~g}$ $\mathrm{cm}^{2}$ Aluminum shield exposed to the February, 1956 Webber SPE.

The fixed-point series method (Slaba, 2007; Heinbockel et al., 2007) is a Neumann series solution to the FB model. The forward and backward components are expressed as

$\phi_{n}^{f}(x, E)=\sum_{k=1}^{\infty} f_{0}^{(k)}(x, E)$

$\phi_{n}^{b}(x, E)=\sum_{k=1}^{\infty} b_{0}^{(k)}(x, E)$

where the $k$ th zero order term satisfies

$B^{(+)}\left[f_{0}^{(k)}\right]=\xi_{k-1}^{f}(x, E)$

$B^{(-)}\left[b_{0}^{(k)}\right]=\xi_{k-1}^{b}(x, E)$,

and for $k>0$ the $k$ th set of source terms are defined

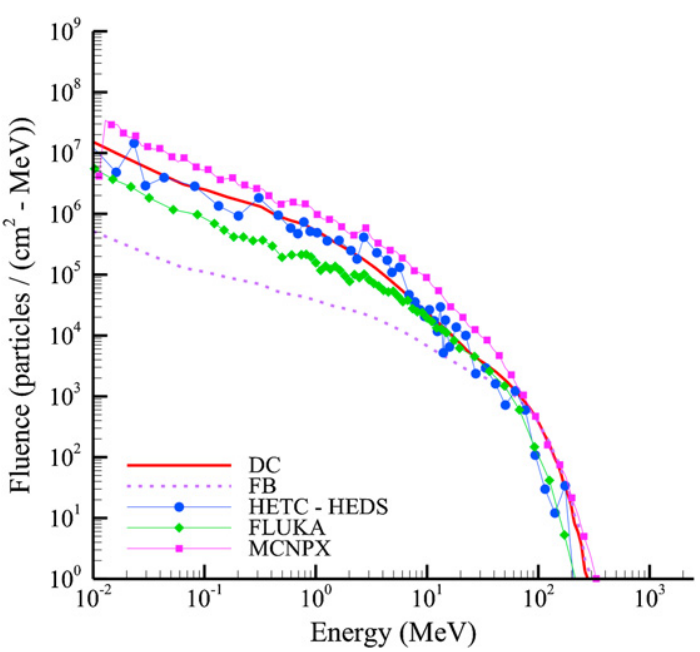

Fig. 6. Backward neutron fluence at $20 \mathrm{~g} / \mathrm{cm}^{2}$ in a $30 \mathrm{~g} / \mathrm{cm}^{2}$ water target behind a $20 \mathrm{~g} /$ $\mathrm{cm}^{2}$ Aluminum shield exposed to the February, 1956 Webber SPE. 
$\xi_{k}^{f}(x, E)=\int_{E}^{\infty} \sigma_{n n}\left(E, E^{\prime}\right) f_{0}^{(k)}\left(x, E^{\prime}\right) \mathrm{d} E^{\prime}$

$\xi_{k}^{b}(x, E)=\int_{E}^{\infty} \sigma_{n n}\left(E, E^{\prime}\right) b_{0}^{(k)}\left(x, E^{\prime}\right) \mathrm{d} E^{\prime}$.

The initial set of source terms $\xi_{0}^{f}(x, E), \xi_{0}^{b}(x, E)$ are simply the forward and backward components of the isotropic neutron source term $\eta_{n}(x, E)$.

Wilson's method (Slaba et al., 2006, 2007; Heinbockel et al., 2007) is a combination of the collocation method and fixed-point series method. The forward and backward neutron fluences are expressed as the sum of a zero order and first order term

$\phi_{n}^{f}(x, E)=f_{0}(x, E)+f_{1}(x, E)$

$\phi_{n}^{b}(x, E)=b_{0}(x, E)+b_{1}(x, E)$.

Equations (57) and (58) are substituted into the FB model, and it is assumed that the zero order terms satisfy

$B^{(+)}\left[f_{0}\right]=\eta_{n}^{f}(x, E)$

$B^{(-)}\left[b_{0}\right]=\eta_{n}^{b}(x, E)$.

The FB model is now reduced to

$B^{(+)}\left[f_{1}\right]=\int_{E}^{\infty} \sigma_{n n}\left(E, E^{\prime}\right) f_{1}\left(x, E^{\prime}\right) \mathrm{d} E^{\prime}+\xi_{1}^{f}(x, E)$

$B^{(-)}\left[b_{1}\right]=\int_{E}^{\infty} \sigma_{n n}\left(E, E^{\prime}\right) b_{1}\left(x, E^{\prime}\right) \mathrm{d} E^{\prime}+\xi_{1}^{b}(x, E)$,

where the source terms $\xi_{1}^{f}(x, E)$ and $\xi_{1}^{b}(x, E)$ are calculated from the zero order terms by

$\xi_{1}^{f}(x, E)=\int_{E}^{\infty} \sigma_{n n}\left(E, E^{\prime}\right) f_{1}\left(x, E^{\prime}\right) \mathrm{d} E^{\prime}$

$\xi_{1}^{b}(x, E)=\int_{E}^{\infty} \sigma_{n n}\left(E, E^{\prime}\right) b_{1}\left(x, E^{\prime}\right) \mathrm{d} E^{\prime}$.

Equations (61) and (62) are solved using the collocation method. Slaba et al. (2006, 2007) and Heinbockel et al. (2007) showed that the three methods produce almost identical results in a variety of shielding configurations exposed to both SPE and GCR environments and compared quite well with the multigroup technique in all cases (Slaba, 2007). The work also revealed that the collocation method was the most computationally efficient of the four methods in terms of both memory requirements and run time. Hereafter, any neutron spectra labeled "FB model" are assumed to be generated using the collocation method.

\section{DC Neutron transport methods}

Feldman (2003) developed a numerical solution for the DC model. A collocation method was used to discretize the energy spectrum as in equations (45) and (46), and first order finite differencing was used to approximate the spatial derivatives and

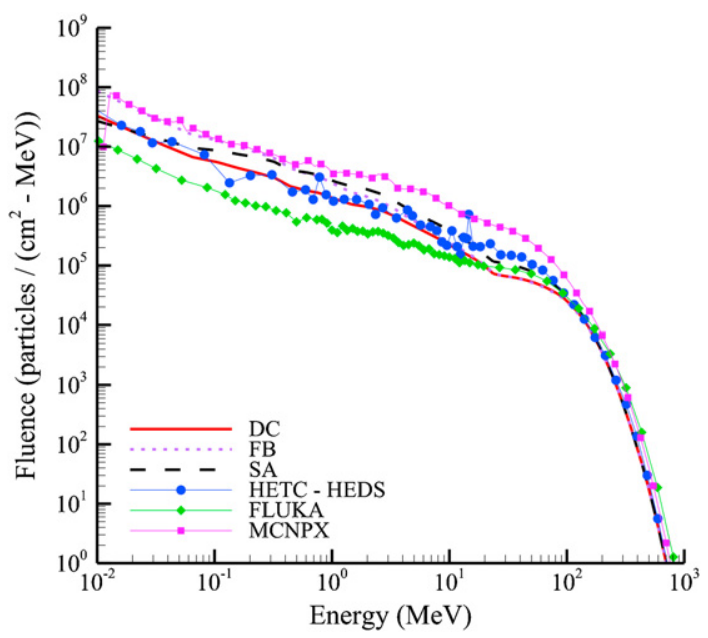

Fig. 7. Total neutron fluence at $20 \mathrm{~g} / \mathrm{cm}^{2}$ in a $30 \mathrm{~g} / \mathrm{cm}^{2}$ water target behind a $20 \mathrm{~g} / \mathrm{cm}^{2}$ Aluminum shield exposed to the February, 1956 Webber SPE.

decouple the transport equations. Discretization of the energy and spatial domains in this way resulted in a large system of linear equations that was solved numerically using LU factorization. LU factorization relies on writing the coefficient matrix as the product of a lower triangular and an upper triangular part so that forward and backward substitution can be used to obtain a solution (Demmel, 1997). It was reported that even single layer slab calculations quickly exhausted the memory capabilities of a desktop PC, and run times of over $20 \mathrm{~h}$ were reported even on specialized computers with adequate memory (Feldman, 2003).

We present here a computationally efficient Neumann series solution to the DC model based on the numerical methods verified for the FB model. The forward and backward components of the isotropic neutron fluence are expressed as a sum of zero order terms

$\phi_{n}^{f}(x, E)=\sum_{k=1}^{\infty} f_{0}^{(k)}(x, E)$

$\phi_{n}^{b}(x, E)=\sum_{k=1}^{\infty} b_{0}^{(k)}(x, E)$

where the $k$ th zero order term satisfies

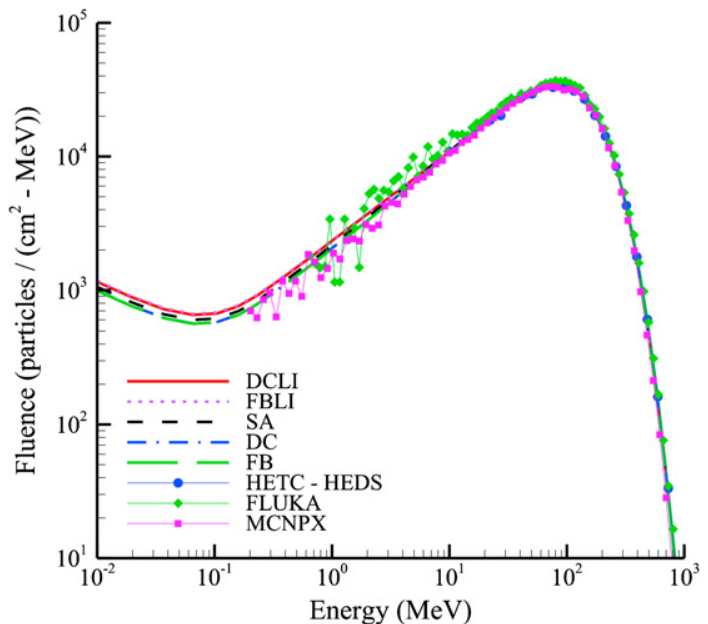

Fig. 8. Proton fluence at $20 \mathrm{~g} / \mathrm{cm}^{2}$ in a $30 \mathrm{~g} / \mathrm{cm}^{2}$ water target behind a $20 \mathrm{~g} / \mathrm{cm}^{2}$ Aluminum shield exposed to the February, 1956 Webber SPE. 
$B^{(+)}\left[f_{0}^{(k)}\right]=\int_{E}^{\infty} \sigma_{n n}^{(+)}\left(E, E^{\prime}\right) f_{0}^{(k)}\left(x, E^{\prime}\right) \mathrm{d} E^{\prime}+\xi_{k-1}^{f}(x, E)$

$B^{(-)}\left[b_{0}^{(k)}\right]=\int_{E}^{\infty} \sigma_{n n}^{(+)}\left(E, E^{\prime}\right) b_{0}^{(k)}\left(x, E^{\prime}\right) \mathrm{d} E^{\prime}+\xi_{k-1}^{b}(x, E)$,

and can be solved using the collocation method outlined previously. Note that solutions to equations (67) and (68) include neutron production with scattering angle between 0 and $\pi / 2$. Neutron production with scattering angle between $\pi / 2$ and $\pi$ is accounted for in the $k$ th set of source terms

$\xi_{k}^{f}(x, E)=\int_{E}^{\infty} \sigma_{n n}^{(-)}\left(E, E^{\prime}\right) b_{0}^{(k)}\left(x, E^{\prime}\right) \mathrm{d} E^{\prime}$

$\xi_{k}^{b}(x, E)=\int_{E}^{\infty} \sigma_{n n}^{(-)}\left(E, E^{\prime}\right) f_{0}^{(k)}\left(x, E^{\prime}\right) \mathrm{d} E^{\prime}$

for $k>0$. The initial set of source terms, $\xi_{0}^{f}(x, E)$ and $\xi_{0}^{b}(x, E)$, are simply the forward and backward components of the isotropic neutron source term $\eta_{n}(x, E)$.

While many terms in the Neumann series are required to adequately resolve the neutron fluence spectra (as many as 75 terms for each component), the computation is quite rapid since the coefficients $a_{i j}$ used in the collocation method and the cross sections appearing in the source terms (equations (69) and (70)) can be pre-calculated and saved for repeated use. No published data exists from Feldman's work, and so any comparisons with data labeled "DC Model" were generated using the proposed Neumann series solution.

\section{Coupling to light ion transport}

In the development of the neutron transport models, we did not solve for the isotropic charged particle fluence

$B\left[\phi_{j}^{i s o}\right]=\sum_{k} \int_{E}^{\infty} \sigma_{j k}\left(E, E^{\prime}\right) \phi_{k}^{i s o}\left(x, E^{\prime}\right) \mathrm{d} E^{\prime}$.

It is important to point out that the summation in equation (71) is taken over light particles only. This is justified by noting that $\phi_{j}^{\text {iso }}$ is associated with low energy target fragments, and heavy ion target fragments are not transported in HZETRN due to their extremely small range (Wilson and Badavi 1986; Wilson et al., 1991, 2006). Their contribution to dose is approximately accounted for after the transport procedure.

The isotropic neutron fluence has already been obtained via one of the bi-directional neutron transport models, and so equation (71) can be expressed in the more explicit form

$B\left[\phi_{j}^{i s o}\right]=\sum_{k \neq n} \int_{E}^{\infty} \sigma_{j k}\left(E, E^{\prime}\right) \phi_{k}^{i s o}\left(x, E^{\prime}\right) \mathrm{d} E^{\prime}+\eta_{j}(x, E)$,

with the source terms

$\eta_{j}(x, E)=\int_{E}^{\infty} \sigma_{j n}\left(E, E^{\prime}\right) \phi_{n}^{i s o}\left(x, E^{\prime}\right) \mathrm{d} E^{\prime}$.

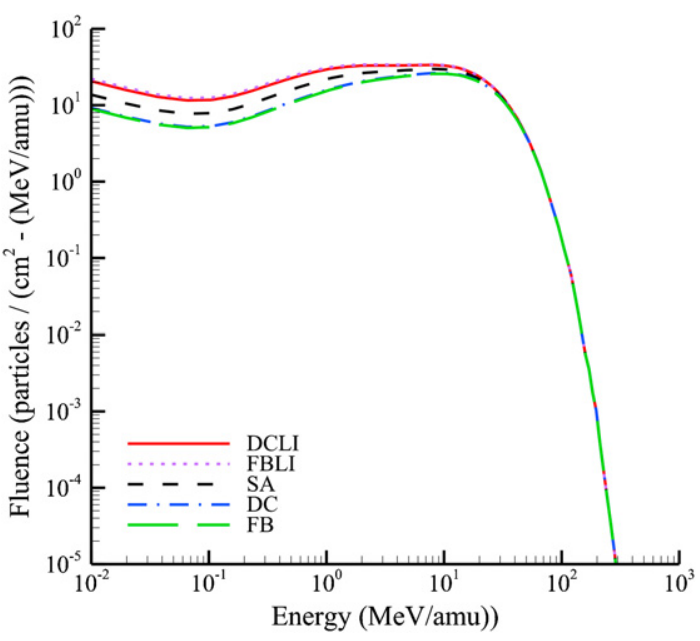

Fig. 9. ${ }^{3} \mathrm{H}$ fluence at $20 \mathrm{~g} / \mathrm{cm}^{2}$ in a $30 \mathrm{~g} / \mathrm{cm}^{2}$ water target behind a $20 \mathrm{~g} / \mathrm{cm}^{2}$ Aluminum shield exposed to the February, 1956 Webber SPE.

We now approximate equation (72) by suppressing the remaining collision integrals. Such an approximation is expected to be quite valid since the nuclear mean free path of these low energy charged particles is much larger than their range (Wilson et al., 1991) as seen earlier in Fig. 4. In this case, equation (72) is now reduced to

$B\left[\phi_{j}^{i s o}\right]=\eta_{j}(x, E)$,

for which an analytic solution exists and is easily found by using the method of characteristics (Haberman, 1998). The solution was given by Wilson and Lamkin (1975) in terms of the nuclear survival probability

$P_{j}(E)=\exp \left[-A_{j} \int_{0}^{E} \frac{\sigma_{j}\left(E^{\prime}\right)}{S_{j}\left(E^{\prime}\right)} \mathrm{d} E^{\prime}\right]$,

and the residual energy $E_{\gamma}=R_{j}^{-1}\left(x+R_{j}(E)\right)$ as

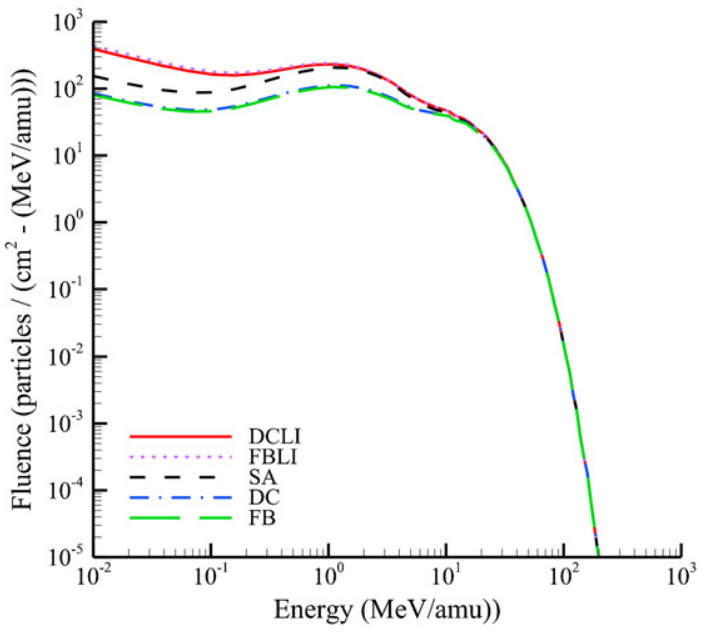

Fig. 10. ${ }^{4} \mathrm{He}$ fluence at $20 \mathrm{~g} / \mathrm{cm}^{2}$ in a $30 \mathrm{~g} / \mathrm{cm}^{2}$ water target behind a $20 \mathrm{~g} / \mathrm{cm}^{2}$ Aluminum shield exposed to the February, 1956 Webber SPE. 
Table 1

Dose (cGy) in a $30 \mathrm{~g} / \mathrm{cm}^{2}$ water target behind $20 \mathrm{~g} / \mathrm{cm}^{2}$ of Aluminum exposed to the February, 1956 Webber SPE spectrum.

\begin{tabular}{lllllll}
\hline $\begin{array}{l}\text { Depth } \\
\mathrm{g} / \mathrm{cm}^{2}\end{array}$ & SA & DCLI & FBLI & DC & FB & HETC-HEDS \\
\hline 0 & 6.97 & 6.99 & 6.98 & 6.95 & 6.93 & 7.20 \\
5 & 3.78 & 3.78 & 3.78 & 3.75 & 3.75 & 4.04 \\
10 & 2.28 & 2.28 & 2.29 & 2.26 & 2.26 & 2.50 \\
20 & 0.98 & 0.98 & 0.99 & 0.96 & 0.97 & 1.10 \\
30 & 0.49 & 0.48 & 0.49 & 0.47 & 0.47 & 0.53 \\
\hline
\end{tabular}

$$
\begin{aligned}
\phi_{j}^{i s o}(x, E) & =\frac{P_{j}\left(E_{\gamma}\right)}{P_{j}(E)} \frac{S_{j}\left(E_{\gamma}\right)}{S_{j}(E)} \phi_{j}^{i s o}\left(0, E_{\gamma}\right) \\
& +\int_{E}^{E_{\gamma}} \frac{A_{j} P_{j}\left(E^{\prime}\right)}{P_{j}(E) S_{j}(E)} \eta_{j}\left(x+R_{j}(E)-R_{j}\left(E^{\prime}\right), E^{\prime}\right) \mathrm{d} E^{\prime},
\end{aligned}
$$

where $R_{j}(E)$ is given by the range energy relation

$$
R_{j}(E)=A_{j} \int_{0}^{E} \frac{\mathrm{d} E^{\prime}}{S_{j}\left(E^{\prime}\right)} .
$$

Equation (76) can be evaluated rapidly using numerical integration schemes and imposes small memory requirements.

\section{Results}

Solutions to equation (1) using the HZETRN marching algorithm will be labeled "SA," and it is assumed that all particles (including neutrons) are transported in the forward direction. Solutions to equation (24) using the HZETRN marching algorithm with FB or DC neutrons added and no light ion coupling will be labeled "FB" or "DC", respectively. Finally, solutions to equation (24) using the HZETRN marching algorithm with FB or DC neutrons and the suggested light ion coupling will be labeled "FBLI" or "DCLI", respectively. The only difference between FB and FBLI or DC and DCLI is the presence of the light ion coupling term given in the previous section. Therefore, neutron fluences predicted by FB and FBLI (or DC and DCLI) will be identical. In all of the neutron fluence comparisons, FB and DC will be used, but could be interchanged with FBLI and DCLI without consequence.

Results are now given at various depths in a $30 \mathrm{~g} / \mathrm{cm}^{2}$ water target behind a $20 \mathrm{~g} / \mathrm{cm}^{2}$ Aluminum shield exposed to the February 1956 Webber SPE spectrum (Quenby and Webber, 1959). The differences between the various Monte Carlo codes has been discussed elsewhere (Heinbockel et al., 2009) and will not be addressed in this paper. Fig. 5 shows the forward component of the neutron fluences at $20 \mathrm{~g} / \mathrm{cm}^{2}$ in the water target. The DCLI model compares quite well to HETC-HEDS and is within the bounds set by FLUKA and MCNPX; the FBLI and SA solutions predict much higher neutron fluences for energies below $100 \mathrm{MeV}$. Fig. 6 gives the backward neutron fluences at $20 \mathrm{~g} / \mathrm{cm}^{2}$ in the water target. The DCLI model again compares quite well to HETC-HEDS and is within the bounds set by FLUKA and MCNPX; it is a significant improvement over the FBLI model. There is no backward component of the SA model since all particles are transported in the forward direction. Finally, Fig. 7 gives the total neutron fluence at $20 \mathrm{~g} / \mathrm{cm}^{2}$ in the water target. As in each of the previous figures, the DCLI model compares well to HETC-HEDS and is within the bounds set by FLUKA and MCNPX. Most importantly, it now appears that HZETRN compares as well to the Monte Carlo codes as they compare with each other.
Table 2

Dose equivalent (cSv) in a $30 \mathrm{~g} / \mathrm{cm}^{2}$ water target behind $20 \mathrm{~g} / \mathrm{cm}^{2}$ of Aluminum exposed to the February, 1956 Webber SPE spectrum.

\begin{tabular}{lrrrrrl}
\hline $\begin{array}{l}\text { Depth } \\
\mathrm{g} / \mathrm{cm}^{2}\end{array}$ & \multicolumn{1}{l}{ SA } & DCLI & \multicolumn{1}{l}{ FBLI } & \multicolumn{1}{l}{ DC } & \multicolumn{1}{l}{ FB } & HETC-HEDS \\
\hline 0 & 11.70 & 11.83 & 11.50 & 11.36 & 11.03 & 8.87 \\
5 & 6.21 & 6.18 & 6.27 & 5.80 & 5.84 & 4.85 \\
10 & 3.94 & 3.87 & 4.01 & 3.56 & 3.69 & 3.03 \\
20 & 1.87 & 1.79 & 1.94 & 1.59 & 1.71 & 1.36 \\
30 & 1.05 & 0.91 & 1.07 & 0.78 & 0.91 & 0.63 \\
\hline
\end{tabular}

Figs. 8-10 give the proton, ${ }^{3} \mathrm{H}$, and ${ }^{4} \mathrm{He}$ fluence spectra generated from the SA, FB, DC, FBLI, and DCLI models at $20 \mathrm{~g} / \mathrm{cm}^{2}$ in the water target. First, note that the spectra predicted by the FB and DC models are identical. This behavior is expected since neither of these models include the suggested light ion coupling, and the charged particle fluences are determined by solutions to equation (24). Next, the spectra predicted by the FBLI and DCLI models are similar but not identical. In fact, the FBLI produced slightly larger light ion fluence spectra than the DCLI model in all three cases. This trend is caused by the light ion coupling source term in equation (72) and the neutron transport model used. Figs. 5-7 showed that neutron fluences obtained with the FB model tended to be larger than the neutron fluences obtained with DC model. Clearly, if the FB results are used, the light ion source term in equation (72) will be larger and so will the isotropic light ion fluences. The FBLI and DCLI light ion spectra agree quite well even though the FB and DC neutron fluences in Fig. 7 are almost an order of magnitude different at the lowest energies. This is not surprising since few charged target fragments are produced from neutrons below $20 \mathrm{MeV}$.

Most importantly, Figs. 9 and 10 show that a significant reduction in low energy fluence spectra can occur if the suggested light ion coupling term is not included. Though little difference is seen in the proton fluence spectra (Fig. 8), the ${ }^{4} \mathrm{He}$ spectrum drops by almost a factor of four if the light ion coupling term is not included. Similar results are also seen for the other light particles transported in HZETRN $\left({ }^{2} \mathrm{H}\right.$ and $\left.{ }^{3} \mathrm{He}\right)$.

Such deviations are significant enough to alter integrated quantities as well. For example, Tables 1 and 2 give the dose and dose equivalent values at various depths in the water target. As expected, these values are all reduced if the light ion coupling is not included. Dose and dose equivalent values were not available from FLUKA and MCNPX at the time of this report.

\section{Conclusions}

A directionally coupled neutron transport model and computationally efficient solution method were presented. Results compared very well with HETC-HEDS and were within the bounds set by FLUKA and MCNPX. A first order approximation was also presented for coupling the neutron models to low energy light ion transport, and results indicated that such a coupling is indeed necessary not only for accurate estimates of low energy fluence spectra, but for integrated quantities such as dose and dose equivalent as well. It is recommended that future studies with HZETRN and bi-directional neutrons use the directionally coupled neutron transport model along with the first order light ion coupling presented here.

\section{Acknowledgements}

This research was sponsored by NASA Research Grant NNLO6AA14A. We would like to thank John W. Wilson for his contributions and guidance in this work. 


\section{References}

Alsmiller, R.G., Irving, D.C., Kinney, W.E., Moran, H.S., 1965. The Validity of the Straightahead Approximation in Space Vehicle Shielding Studies. Second Symposium on Protection Against Radiations in Space. NASA SP 71.

Briesmeister, J.F. (Ed.), 2000. MCNP-A General Monte Carlo N-Particle Transport Code-Version 4C, Los Alamos National Laboratory Report LA-13709-M.

Clowdsley, M.S., Heinbockel, J.H., Kaneko, H., Wilson, J.W., Singleterry, R.C., Shinn, J.L., 2000a. A comparison of the multigroup and collocation methods for solving the low energy neutron Boltzmann equation. Canadian Journal of Physics 78, 45-56.

Clowdsley, M.S., Wilson, J.W., Heinbockel, J.H., Tripathi, R.K., Singleterry, R.C., Shinn, J.L., 2000b. An Improved elastic and nonelastic neutron transport algorithm for space radiation. NASA Technical Paper 210299.

Cucinotta, F.A., 1993. Calculations of cosmic-ray helium transport in shielding materials. NASA Technical Paper 3354.

Demmel, J.W., 1997. Applied Numerical Linear Algebra. Society for Industrial and Applied Mathematics, Philadelphia.

Deuflhard, P., Bornemann, F., 2002. Scientific Computing with Ordinary Differential Equations. Springer, New York.

Fasso, A., Ferrari, A., Roesler, S., Sala, P.R., Battistoni, G., Cerutti, F., Gadioli, E., Garzelli, M.V., Ballarini, F., Ottolenghi, A., Empl, A., Ranft, J., 2003. The physics models of FLUKA: status and recent developments. In: Computing in High Energy and Nuclear Physics 2003 Conference (CHEP2003), La Jolla, CA, March 24-28, 2003 (paper MOMT005), eConf C0303241 (2003), arXiv:hep-ph/0206267.

Fasso, A., Ferrari, A., Ranft, J., Sala, P.R., 2005. Fluka: A Multi-Particle Transport Code. CERN-2005-10, INFN/TC_05/11, SLAC-R-773.

Feldman, G., 2003. A Forward Backward Fluence Model for the Low Energy Neutron Boltzmann Equation. Ph.D. thesis, Old Dominion University.

Gabriel, T.A., Bishop, B.L., Alsmiller, F.S., Alsmiller, R.G., Johnson, J.O., 1995. Colar95: A Monte Carlo Program Package for the Design and Analysis of Calorimeter Systems. ORNL. TM-11185.

Getselev, I., Rumin, S., Sobolevsky, N., Ufimtsev, M., Podzolko, M., 2004. Abosrbed dose of secondary neutrons from galactic cosmic rays inside the international space station. Advances in Space Research 34, 1429-1432.

Haberman, R., 1998. Elementary Applied Partial Differential Equations. Prentice Hall, New Jersey.

Haffner, J.W., 1967. Radiation and Shielding in Space. Academic Press, New York.

Heinbockel, J.H., Clowdsley, M.S., Wilson, J.W., 2000. An improved neutron transport algorithm for space radiation. NASA Technical Paper 209865.

Heinbockel, J.H., Slaba, T.C., Wilson, J.W., Blattnig, S.R., Clowdsley, M.S., Badavi, F.F., 2007. Comparison of Numerical Solution Techniques for Calculating Low Energy Neutrons. SAE. ICES 2007013117.
Heinbockel, J.H., Slaba, T.C., Blattnig, S.R., Tripathi, R.K., Townsend, L.W., Handler, T. Gabriel, T.A., Pinsky, L.S., Reddell, B., Clowdsley, M.S., Singleterry, R.C. Norbury, J.W., Badavi, F.F., Aghara, S.K., 2009. Comparison of the radiation transport codes HZETRN, HETC-HEDS and FLUKA using the February 1956 webber SPE spectrum. NASA Technical Paper 2009, 215560.

Marchuk, G.I., Lebedev, V.I., 1986. Numerical Methods in the Theory of Neutron Transport. Harwood Academic Publishers, New York.

MCNPX 2.6.0, April, 2008. Manual, Los Alamos Report LA-CP-07-1473, Los Alamos.

Quenby, J.J., Webber, W.R., 1959. Cosmic ray cut-off rigidities and the earth's magnetic field. Philosophical Magazine 4, 90-112.

Ranft, J., 1980. The FLUKA and KASPRO Hadronic Cascade Codes. Computer Techniques in Radiation Transport and Dosimetry. Plenum Press, pp. 339-371.

Shinn, J.L., Wilson, J.W., Weyland, M., Cucinotta, F.A., 1991. Improvements in computational accuracy of BRYNTRN (A Baryon transport code). NASA Technical Paper 3093.

Shinn, J.L., Wilson, J.W., Lone, M.A., Wong, P.Y., Costen, R.C., 1994. Preliminary estimates of nucleon fluxes in a water target exposed to solar flare protons: BRYNTRN versus Monte Carlo code. NASA Technical Memorandum 4565.

Slaba, T.C., Heinbockel, J.H., Wilson, J.W., Blattnig, S.R., Clowdsley, M.S., Badavi, F.F. 2006. A New Method for Calculating Low Energy Neutron Flux. SAE. ICES 2006 012149.

Slaba, T.C., 2007. Three Methods for Solving the Low Energy Neutron Boltzmann Equation. Ph.D. thesis, Old Dominion University.

Townsend, L.W., Miller, T.M., Gabriel, T.A., 2005. HETC radiation transport code development for cosmic ray shielding applications in space. Radiation Protection Dosimetry 115, 135-139.

Wilson, J.W., Lamkin, S.L., 1975. Perturbation theory for charged-particle transport in one dimension. Nuclear Science and Engineering 57, 292-299.

Wilson, J.W., Badavi, F.F., 1986. Methods of galactic heavy ion transport. Radiation Research 108, 231-237.

Wilson, J.W., Townsend, L.W., Schimmerling, W., Khandelwal, G.S., Khan, F., Nealy, J.E., Cucinotta, F.A., Simonsen, L.C., Shinn, J.L., Norbury, J.W., 1991 Transport methods and interactions for space radiations. NASA Reference Publication 1257.

Wilson, J.W., Korte, J.J., Sobieski, J., Badavi, F.F., Chokshi, S.M., Martinovic, Z.N., Cerro, J., Qualls, G.D., 2003. Radiation shielding, MDO processes, and single stage to orbit design. In: AIAA Space 2003 Conference, AIAA 20036259.

Wilson, J.W., Tripathi, R.K., Mertens, C.J., Blattnig, S.R., Clowdsley, M.S. Cucinotta, F.A., Tweed, J., Heinbockel, J.H., Walker, S.A., Nealy, J.E., 2005. Verification and validation: high charge and energy (HZE) transport codes and future development. NASA Technical Paper 213784.

Wilson, J.W., Tripathi, R.K., Badavi, F.F., Cucinotta, F.A., 2006. Standardized Radiation Shield Design Method: 2005 HZETRN. SAE. ICES 200618. 